\title{
Integrating th the intellectual property value chain
}

Biotechnology companies can use "value-chain analysis" to create processes for maximizing the value of their intellectual property portfolios, say Bill Barrett and Dave Orawford.

\section{very biotechnology company begins as an idea. With an invest- ment of time and money-and not a little luck - the successful} entrepreneur can develop and execute a strategy for generating revenue from that idea. Inevitably, entrepreneurs must focus their time and attention on obtaining investment capital and building a profitable business model. Viewed al ongside these primary objec-

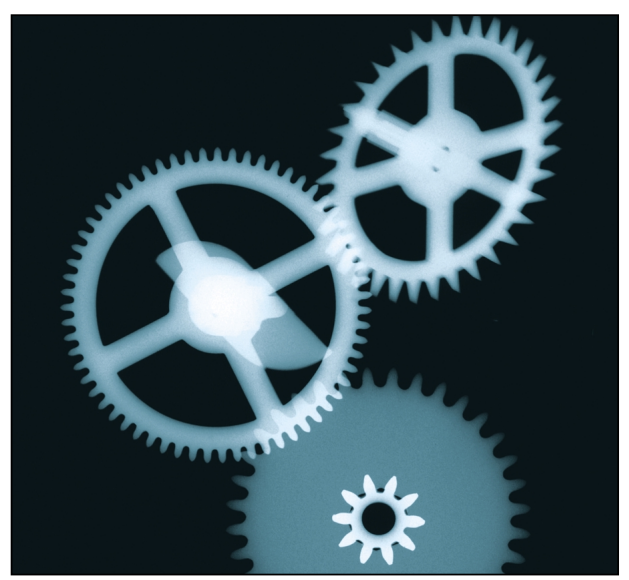
tives, the long-term, strategic importance of intellectual property (IP) can fade into the background. (In this article we use the term "intellectual property" in a narrow sense, to refer to intangible property that relates to inventions, such as patents and trade secrets.) For a biotechnology company, however, a strong IP portfolio is a determinant of success: IP supports future revenue streams and erects barriers to competition, and its quality is intimately tied up with the company's perceived value to investors, partners, and acquirers.

However, building an IP portfolio can be a large expense for a young company operating on limited investment capital. Because of the crucial role of IP in a company's potential value, companies need to ensure that their investment in IP will yield a strategically targeted IP portfolio.

Common reasons why companies fail in

Bill Barrett and Dave Crawford areintellectual property consultants at ipCapital Group, Williston, VT (bbarrett@ipcapitalgroup.com; dcrawford@ipcapitalgroup.com). tures and the business strategy;

- Lack of institutional knowledge of IP concepts and tactics;

- Lack of internal processes for extracting, evaluating, and capitalizing on IP.

To solve these problems, we find it helpful to think of a company's IP processes in terms of a "value chain." In general, a value chain is a series of steps in which each new step adds incremental value to the one before. The IP value chain starts with the inventor's original idea and has value added by a series of steps that ultimately yields a legally protected asset (e.g., a patent or a trade secret).

\section{Typical IP value chain}

Without a concerted effort to develop an integrated IP value chain, most companies will instinctively follow a somewhat nonintegrated approach to the creation of an IP portfolio (Fig. 1). Conception of the original idea is followed by its documentation in laboratory notebooks and/or electronic files. Inventions are then reviewed informally, and those believed to have value may be formally documented in "invention disclosure documents." Disclosure documents are then delivered to patent counsel for legal documentation (such as the preparation of a patent application) and patent prosecution. Although the value steps flow into one another, the process is passive, lacks true integration, and is not directed by the business strategy. As a result, the chain can produce an IP portfolio that is misaligned with the direction in which the company is moving.

\section{Integrated IP value chain}

Companies can address the limitations of the typical IP value chain by treating IP as a manageable asset and by incorporating it into the business planning process much 
(C) Bob Crimi

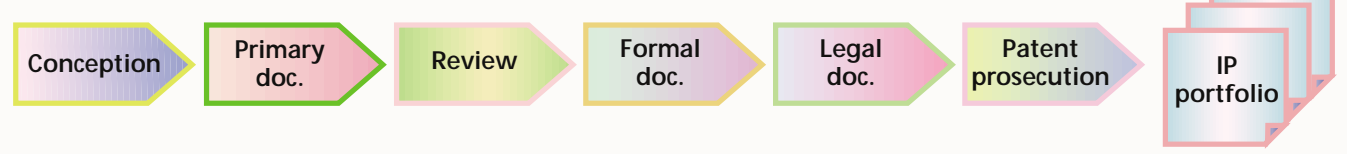

Conception. Because inventors are intimately involved with the finer details of the thousands of incremental steps leading up to their invention, they often find it difficult to

Figure 1. Value chain that naturally evolves in companies lacking a proactive, integrated IP value chain. gain a perspective on how much the final "invention"

like any other business process. The result is a fully integrated IP value chain (Fig. 2) that helps the company to build a targeted portfolio of IP that supports its business objectives.

Building a foundation. The foundation of the IP value chain includes business strategy, competitive IP intelligence, the IP roadmap, and education. These components support and direct each of the steps of the IP value chain, and they must all be in place for the company to derive maximum benefit from it.

Business strategy. A comprehensive business strategy must guide and direct the IP value chain. The business strategy must set clear top-level IP objectives for the organization. For example, assume that you are the chief executive officer of N ewco, a startup based on the discovery of an attenuated adeno-associated virus that can deliver genetic material to lung cells with high transfection efficiency. Your technology solves one of the key problems plaguing efforts to devel op a gene therapy for cystic fibrosis. Your business strategy may involve devel oping the methods required for mass production, storage, distribution, and clinical administration of your attenuated virus, and the direct sale of kits and reagents for its administration in the clinic. To successfully real ize this strategy, you may be committed to protecting its path to market by seeking global patent protection across your entire product and service space, including specialized reagents, production methods, intermediates, storage devices and methods, business processes related to sale and distribution, and methods and kits for clinical use.

Competitive IP intelligence. The business strategy is informed by competitive IP intelligence, which establishes the basic features of your patent landscape. We are often surprised by how uninformed many companies are about their competitors' $I P$, even though the competitors' patents and patent publications are publicly available and can be easily identified through the Internet. A detailed understanding of the competitive patent landscape must take into account not only the patents of direct competitors, but also patents of sup- pliers and customers (and their competitors) and companies in related technology areas. Understanding the patent landscape can help a company avoid the mistake of forging into a heavily patented area, or at least permit the company to develop a rational strategy if it chooses to work in a competitive arena. In addition, the patent literature can provide insight into the priorities and strategies of competitors, and point to potential acquirers, licensors of technology, and targets for merger and acquisition activity.

IP roadmap. Once the competitive IP landscape has been established, the company can develop an IP roadmap - a stepby-step plan that outlines the finer details of the IP strategy and directs decisions about IP investment. The map points inventors to the areas where they should innovate and the legal team to what it should patent. The IP roadmap also maps out other IP tactics, such as the use of defensive publications to weaken a competitor's IP position? ${ }^{1}$.

Education. The final foundation stone of the IP value chain is education. Education ensures that those participating in the process understand how all the steps- the roadmap, strategy, and intelligence- fit together in the value chain, and therefore what is expected of them. For example, when inventors understand the relationship between patent scope and "enablement requirement" (i.e., the requirement that the patent describe the invention in such terms that a person skilled in the relevant art could make and use the invention), they can design research protocols that will support broader enablement and thus broader patent scope.

\section{Steps of the integrated IP value chain}

With the proper foundations in place, the steps of the integrated IP value chain can work together like a smoothly operating machine to generate IP. These steps include conception, primary documentation, capture, initial review, formal documentation, formal review, legal documentation, and patent prosecution (Fig. 2). actually differs from the previous state of the art. As a result, inventors often mentally discard inventions with significant value before they are identified and documented in a manner that permits a formal assessment of their potential value. Companies can solve this problem by ensuring that inventors are trained to recognize inventions that might be patentable. For example, inventors should understand the basic requirements for patentability of an invention: novelty, non-obviousness, enablement, and embodiment of patentable subject matter. Inventors should also be made aware of other factors that help to build a strong case for patentability, such as unexpected synergistic effects of a combination of known elements (for example, the synergistic effects of using multiple drugs to treat a disease), instances in which published references discourage the combination, or other surprising effects of the combination (such as the reduced side-effects of a drug cocktail).

D ocumentation. Documentation is at the heart of the creation and management of IP. If an invention is to make its way to the patent office, a formal patent application that is adequate for legal purposes must be prepared. However, a huge gap separates an invention at conception from one described in a patent application. In the IP value chain, this "gap" is filled by primary documentation, invention capture, and formal documentation.

Implementation of these steps is the difference between proactive IP management and reactive management of IP that just happens to "crop up."

Primary documentation. Primary documentation includes laboratory notebooks, spreadsheets, and other data relating to the enablement of the invention. H owever, primary documentation is not suitable for tracking and managing inventions. Key information about a single invention is often distributed among various forms of primary documentation and in the inventors' minds. Laboratory notebooks ordinarily contain large amounts of extraneous information that must be waded through to identify the invention. Inventions can therefore becomestuck within primary 


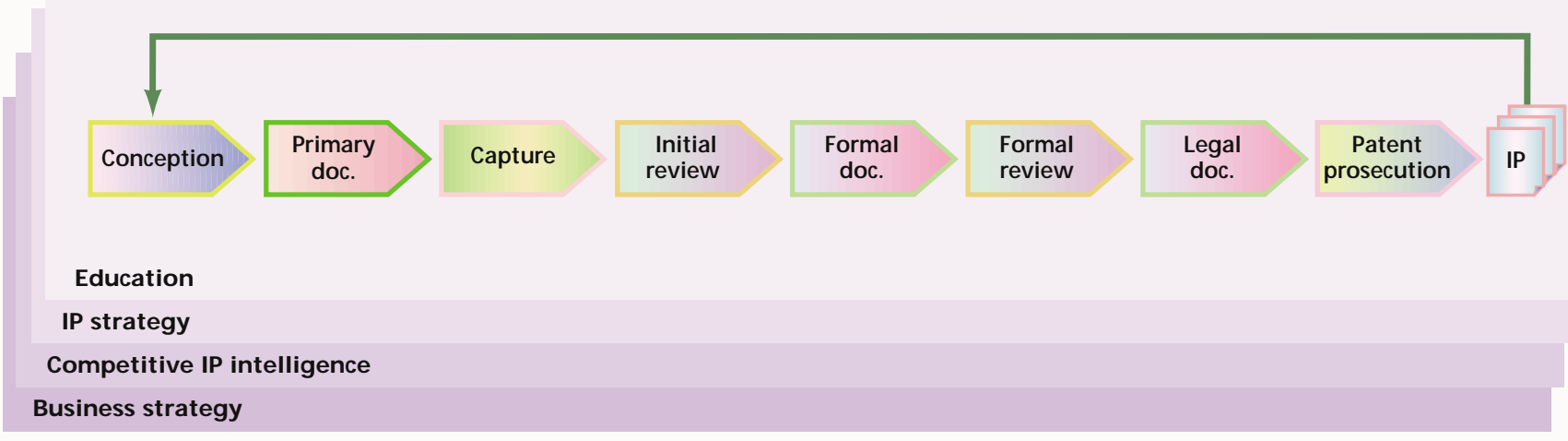

Figure 2. The integrated IP value chain, showing its foundations of business strategy, competitive IP strategy, and education, their integration into the IP value chain, and the feedback loop.

documents because they are virtually invisible to management. The integrated IP value chain solves this problem by providing an intermediate capture step between primary documentation and formal documentation.

Capture. Capture involves actively gathering key information about inventions from primary documentation and from the minds of the inventors, and then placing all this information in a centralized database. Each invention is briefly documented, along with parameters such as preparing formal disclosures for inventions that management does not consider valuable enough to pursue.

Formal documentation. $M$ any companies lack a formal process, standards, and training for the preparation of invention disclosure documents. For this reason, the quality of invention disclosures often varies widely, and invention disclosures lack key details needed by patent counsel and management to understand and evaluate inventions. Thelack or poor quality of invention disclosures increases the time

\section{Legal, technical, and business team members work together to evaluate invention disclosures and make decisions about how to protect each invention.}

priority and stage of development. By lowering the bar for initial documentation (relative to a formal invention disclosure document), the capture step ensures that virtually all potentially patentable or trade secret inventions are documented in a form that permits them to bereviewed and managed.

Initial review. Once inventions are captured, an initial decision must be made regarding which of the inventions will be fleshed out in formal "invention disclosure" documents. This review step typically involves a small committee including technical, legal, and business representatives.

The decision is informed by the IP roadmap: that is, any inventions that could conceivably be aligned with the IP roadmap should be documented for further consideration. The initial review step ensures that inventors do not waste time and effort required for the patent counsel to prepare a patent application, increases the cost of each patent application, and may even reduce the number of patents that a company can afford to file.

The primary component of the formal documentation is a detailed description of the invention. The description should contain a level of detail that would permit another scientist skilled in the area to replicate the invention and use it. Additionally, the document should include other information that can help the company make an informed decision about whether to invest in the invention.

Examples include the next steps required to val idate the concept; issues relating to its commercial viability, such as the costs of manufacturing in bulk; how the invention helps the company capture steps in its value chain; and information about related inventions and patents known to the inventors.

Formal review. In most companies using invention disclosures, the documents tend to bouncefrom person to person and then out the door to the patent counsel, who reviews the disclosures and may then schedule a meeting with the inventor(s) to discuss the invention in more detail. When invention disclosures are bounced around in this fashion, an opportunity for collaboration between legal, technical, and business experts is lost, resulting in a diminished quality of the resulting IP portfolio.

In the enhanced IP value chain, we recommend a formal review step in which legal, technical, and business team members work together to evaluate invention disclosures and make decisions about how to protect each invention in light of the IP roadmap and the business strategy. Strategies for protection generally include patenting the invention, maintaining it as a trade secret, or disclosing it in a defensive publication (defensive publications become so-called prior art and can be used to block or narrow the claims of a competitor's patent). The members of the multidisciplinary team play specific roles: the technical member(s), including the inventor, discuss technical issues and help the legal and business team members to understand how the invention is technically distinct from the state of the art (its novelty and non-obviousness). The legal team member(s) discuss legal issues relating to patentability and probable scope of patent claims. The business team member(s) discuss the business strategy and analyze whether the probable scope of the patent claims justifies the investment. The review process ensures that patents are pursued only where they are jus- 
tified by the business strategy, minimizing the costly pursuit of IP that does not support specific business objectives. Proactive collaboration allows the management team, legal team, and the inventor to create the highest-value IP.

Legal documentation and patent prosecution. Should the formal review process result in a decision to patent an invention, the invention disclosure is communicated to patent counsel for legal documentation and patent prosecution. $M$ anagers and inventors are often discouraged by the legalese of patent applications, especially patent claims, and fail to review them carefully during patent preparation and prosecution. This can result in a patent whose scope does not meet the company's expectations. Patent claims are the most important component of the patent application, and the input of a technical expert is required to ensure that they achieve their greatest value. Companies should invest in educating inventors and business leaders to read and understand claims to ensure that patents have scope and content that is in line with their strategic needs.
Feedback. One of the most important facets of the enhanced IP value chain is the feedback loop. Feedback takes place as the IP roadmap is executed to build a strategically targeted IP portfolio. Updating the IP roadmap with information about the growing IP portfolio ensures that all the players can understand how the company is performing against its IP and business objectives and where investment or management attention is required. Feedback allows management to identify weaknesses in the patents and prompts inventor(s) to identify potential improvements. Fundamentally, through the feedback loop, a company constantly revises its strategy and roadmap in the light of its IP, and this in turn guides what is invented and how this IP is handled. Feedback is therefore an extremely powerful process that can give a company a significant competitive advantage in the marketplace.

\section{Summary}

Building an integrated and fully supported IP value chain offers a number of substantial advantages. First, by standardizing the steps of IP devel opment, companies can reduce wasted effort and streamline IP development, thereby reducing its cost per unit. Second, companies can improve their IP portfolios both quantitatively and qualitatively, capture the maximum amount of potential IP, develop a portfolio that is aligned with specific business goals, and eliminate IP that does not support future revenue streams. Finally, companies can increase the speed of IP development by directing inventors to work on the most important problems.

Building an enhanced IP value chain requires an effort similar to that required for the development of any core business process. For companies whose survival is determined by the quality of their inventions, careful attention to the development of an enhanced IP value chain is essential to maximizing the value of every idea and creating the greatest possible value for shareholders.

1. Barrett, B. Defensive use of publications in an intellectual property strategy. Nat. Biotechnol. 20, 191-193 (2002). 